\title{
Comparação das alterações do potencial evocado somatosensorial no tratamento cirúrgico da escoliose idiopática entre técnicas com e sem amarrilha sublaminar
}

Comparison of the somatosensory evoked potentials changes in the surgical treatment of idiopathic scoliosis between two techniques with and without sublaminar wiring

Comparación de las alteraciones del potencial evocado somatosensorial en el tratamiento quirúrgico de la escoliosis idiopática entre técnicas con y sin amarilla sublaminar

\author{
Renato Hiroshi Salvioni Ueta' \\ David Del Curto' \\ Marcelo Wajchenberg ${ }^{2}$ \\ Délio Eulálio Martins Filho² \\ Ricardo Ferreira ${ }^{2}$ \\ Eduardo Barros Puertas ${ }^{3}$
}

\section{RESUMO}

Objetivo: comparar o número de eventos com alteração no potencial evocado somatosensorial (PESS) e sua repercussão entre técnicas de tratamento cirúrgico da escoliose idiopática com e sem amarrilha sublaminar. Métodos: análise retrospectiva de 25 cirurgias de correção de escoliose idiopática, flexíveis, realizadas no período de novembro de 1996 a setembro de 1999 , nas quais foram utilizadas técnicas sem amarrilha sublaminar (sistema de CotrelDubousset) (Grupo I) e com amarrilha (sistema de Harrington-Luque e haste de Hartshill) (Grupo II). Todos os procedimentos foram realizados com monitoração neurofisiológica da função medular através do PESS. Resultados: no Grupo II, foi obser-

\section{ABSTRACT}

Objective: to compare the number of events with alteration in the somatosensory evoked potentials (SSEP) and its repercussion between different techniques of surgical treatment for idiopathic scoliosis, with and without sublaminar wiring. Methods: twenty-five surgical procedures with flexible curves for treatment of idiopathic scoliosis were reviewed in the period of November 1996 to September 1999. They were divided into two groups: without sublaminar wiring (CotrelDubousset's system) (Group I); and with sublaminar wiring (HarringtonLuque's system and rectangle of Hartshill) (Group II). In all surgeries, the intraoperative neurophysiologic monitoring with Somatosensory
\end{abstract}

\section{RESUMEN}

Objetivo: comparar el número de eventos con alteración en el potencial evocado somatosensorial (PESS) y su repercusión entre técnicas de tratamiento quirúrgico de la escoliosis idiopática, con y sin amarilla sublaminar. Métodos: análisis retrospectivo de 25 cirugías de corrección de escoliosis idiopática, flexibles, realizadas en el periodo de noviembre de 1996 a septiembre de 1999, en las cuales fueron utilizadas técnicas sin amarilla sublaminar (sistema de Cotrel-Dubousset) (Grupo I) y con amarilla (sistema de Harrington-Luque y asta de Hartshill) (Grupo II). Todas las cirugías fueron realizadas con monitorización neurofisiológica de la función medular por medio de PESS. Resultados: en el Grupo II fue obser-

\footnotetext{
Trabalho realizado no Hospital São Paulo da Escola Paulista de Medicina - Universidade Federal de São Paulo - UNIFESP - São Paulo (SP), Brasil.

'Médico Estagiário do Grupo da Coluna do Departamento de Ortopedia e Traumatologia da Escola Paulista de Medicina da Universidade Federal de São Paulo UNIFESP - São Paulo (SP), Brasil.

${ }^{2}$ Médico Assistente do Grupo da Coluna do Departamento de Ortopedia e Traumatologia da Escola Paulista de Medicina da Universidade Federal de São Paulo UNIFESP - São Paulo (SP), Brasil.

${ }_{3}^{3}$ Professor Associado; Chefe do Grupo da Coluna do Departamento de Ortopedia e Traumatologia da Escola Paulista de Medicina da Universidade Federal de São Paulo - UNIFESP - São Paulo (SP), Brasil. 
vada uma frequência maior de alterações do PESS tanto na amplitude como na latência da onda, durante e ao final da cirurgia. Na série revista, constatou-se uma elevada porcentagem de resultados falso-positivos. Em nenhum paciente foi observada qualquer alteração neurológica no pós-operatório. Conclusão: permanece em aberto qual o método mais seguro para o tratamento cirúrgico da escoliose idiopática. Os resultados apresentados neste trabalho sugerem uma menor incidência de alterações na monitoração neurofisiológica da medula em pacientes tratados pelo sistema de Cotrel-Dubousset.
Evoked Potentials (SSEPS) was used. Results: according to the findings, a bigger frequency of monitoring changes was observed during and at the end of the surgery in Group II. A high incidence of false-negative changes was also verified. No patient with neurological damages was observed. Conclusion: There are still doubts about the safest method for the surgical treatment of idiopathic scoliosis. The results presented in this study suggest a smaller incidence of SSEPS changes in the patients treated with Cotrel-Dubousset's system.

KEYWORDS: Scoliosis; Evoked potentials, somatosensory; Surgical procedures, operative; Orthopedic fixation devices vada una frecuencia mayor de alteraciones del PESS, tanto en la amplitud como en la latencia de onda, durante y al final de la cirugía. En la serie revista, se constató un elevado porcentaje de resultados falsos-positivos. En ningún paciente fue observada cualquier alteración neurológica en el postoperatorio. Conclusión: está en discusión cuál es el método más seguro para el tratamiento quirúrgico de la escoliosis idiopática. Los resultados presentados en este trabajo sugieren una menor incidencia de alteraciones en la monitorización neurofisiológica de la médula en los pacientes tratados por el sistema de Cotrel-Dubousset.

DESCRIPTORES: Escoliosis; Potenciales evocados somatosensoriales; Procedimientos quirúrgicos operativos; Dispositivos de fijación ortopédica

\section{INTRODUÇÃO}

A escoliose idiopática é definida como uma curvatura da coluna no plano coronal que ocorre em crianças sem nenhuma causa estabelecida ${ }^{1}$. Correção cirúrgica e estabilização (artrodese) por meio de técnicas cirúrgicas ortopédicas têm sido empregadas como único tratamento para os casos severos.

A incidência de complicações neurológicas decorrentes do tratamento cirúrgico desta doença, incluindo paraplegia completa ou parcial, tem sido reportada em até $0,72 \%$ dos $\operatorname{casos}^{2,3}$. Para prevenir tal complicação, algumas formas de avaliação da função medular durante a cirurgia foram criadas.

O modo mais antigo para avaliar a função medular é a realização do teste do despertar (teste de Stagnara). Para isto, é necessário interromper o ato operatório para permitir que o paciente realize o teste ${ }^{4,5}$, não podendo ser repetido por muitas vezes. O potencial evocado somatosensorial (PESS) foi desenvolvido para permitir aos cirurgiões identificar precocemente momentos potenciais de lesão.

O potencial evocado somatosensorial é a resposta do cérebro a um estímulo aplicado. Estudos experimentais demonstraram que um dano neurológico diminui o PESS, causando uma diminuição da amplitude de onda e aumento de sua latência. De modo padrão, a análise do PESS (amplitude e latência da sua onda) inicia-se antes da incisão, obtendo-se um valor basal. Uma diminuição maior que $50 \%$ da amplitude ou um aumento maior que $10 \%$ da latência correspondem a uma resposta anormal ${ }^{4,6}$.

O objetivo deste estudo foi avaliar a quantidade de alterações no PESS entre diferentes cirurgias para correção de escoliose idiopática, com comparação entre procedimentos nos quais foram utilizados o sistema de CotrelDubousset (ganchos e parafusos pediculares) e sistemas que utilizam hastes com amarrilha (Harrington-Luque e Hartshill).

\section{MÉTODOS}

Estudo retrospectivo com 25 pacientes, média de idade de 14,6 anos (10 a 19 anos), diagnosticados com escoliose idiopática, operadas no período de novembro de 1996 a setembro de 1999, sendo que em 9 pacientes foi utilizado o sistema de Cotrel-Dubousset (Grupo I), em 11 o sistema de Harrington-Luque (Grupo II), e em 5 a haste de Hartshill (Grupo II) (Tabela 1).

Em todos os pacientes analisados, foi realizada a monitoração neurofisiológica da função medular por meio da avaliação do potencial evocado somato-sensitiva. Foram comparados os resultados obtidos na pré e pós-indução anestésica, no intraoperatório e no pós-operatório. O PESS realizado nos membros superiores foi obtido com a estimulação do nervo mediano no nível dos punhos e nos membros inferiores com a estimulação do nervo tibial no nível dos tornozelos, todos na frequência de 3 /segundo. 
As alterações consideradas anormais, como a diminuição da amplitude maior que $50 \%$ e o aumento da latência em mais de $10 \%$ (neste estudo considerado um valor maior de $3 \mathrm{~ms}$ ), foram comunicadas imediatamente ao cirurgião. Realizavam-se, então, interrupções no ato cirúrgico.

O exame físico neurológico foi realizado no pré-operatório e no pós-operatório de todos os pacientes.

\section{RESULTADOS}

Dos 9 pacientes tratados com o sistema de Cotrel-Dubouset, em 4 (44,4\%) foram observadas diminuições maiores que $50 \%$ da amplitude da onda do PESS, sendo que em um deles o sinal foi abolido. Diminuições maiores de 50\% foram observadas em todos os pacientes tratados com o sistema de Harrington-Luque (11 pacientes) e em 3 destes houve uma ausência de resposta. Por consequência, neste grupo foi observado o maior número de interrupções, com tempo médio de 24 minutos, até ser obtido ao menos

TABELA 1 - Distribuição dos grupos

\begin{tabular}{ccc}
\hline & $\begin{array}{c}\text { Grupo I (Cotrel- } \\
\text { Dubousset) }\end{array}$ & $\begin{array}{c}\text { Grupo II (Harrington- } \\
\text { Luque e Hartshill) }\end{array}$ \\
\hline Total & 9 & 16 \\
\hline
\end{tabular}

$50 \%$ dos valores iniciais. Entre os pacientes tratados com haste de Hartshill (5 pacientes), 3 (60\%) apresentaram sinal anormal da amplitude, sendo que em 1 deles $(20 \%)$ foi observada a ausência de resposta. No total, $87,5 \%$ dos pacientes do Grupo II apresentaram, em algum momento, alteração na resposta do PESS (Tabelas 2 e 3).

Quanto ao número de eventos nos quais houve alterações na amplitude do PESS, no Grupo I ocorreu uma média de 0,7 evento por cirurgia, enquanto no Grupo II o valor aumentou para 1,6 evento por cirurgia.

Em relação à latência de onda, não foram observadas alterações significativas nos pacientes nos quais foram utilizados o sistema de Cotrel-Dubousset; todavia, em $36 \%$ e em $20 \%$ dos pacientes tratados com sistema de Harrington-Luque e haste de Hartshill, respectivamente, houve uma latência alterada, isto é, maior que $3 \mathrm{~ms}$. Com isto, em $31,2 \%$ dos pacientes do Grupo II, alguma resposta alterada da latência de onda foi observada no

TABELA 2 - Variação da amplitude de onda no intraoperatório por grupo

\begin{tabular}{lcccc}
\hline & $<50 \%$ & $>50 \%$ & Abolido & Sem alterações \\
\hline Grupo I & 4 & 3 & 1 & 1 \\
Grupo II & 2 & 10 & 4 & 0 \\
\hline
\end{tabular}

TABELA 3 - Número de eventos com alterações de resposta no PESS separados por ato operatório

\begin{tabular}{|c|c|c|c|c|c|c|c|c|}
\hline \multirow{2}{*}{ Paciente } & \multirow{2}{*}{ Idade } & \multirow{2}{*}{ Sexo } & \multirow{2}{*}{ Instrumental } & \multicolumn{3}{|c|}{ Variação da amplitude } & \multicolumn{2}{|c|}{ Variação da latência } \\
\hline & & & & $<50 \%$ & $>50 \%$ & Abolido & $<3 \mathrm{~ms}$ & $>3 \mathrm{~ms}$ \\
\hline VP & 12 & $\mathrm{~F}$ & $C D$ & 1 & 2 & 1 & 1 & 0 \\
\hline MPSP & 15 & M & $C D$ & 1 & 2 & 0 & 1 & 0 \\
\hline CMS & 18 & $\mathrm{~F}$ & $\mathrm{HL}$ & 4 & 2 & 3 & 1 & 3 \\
\hline ALPS & 11 & $\mathrm{~F}$ & HT & 0 & 2 & 0 & 0 & 2 \\
\hline AK & 13 & $\mathrm{~F}$ & $\mathrm{HL}$ & 1 & 0 & 0 & 0 & 0 \\
\hline DCM & 13 & $\mathrm{~F}$ & $C D$ & 1 & 0 & 0 & 0 & 0 \\
\hline DGM & 12 & $\mathrm{~F}$ & $C D$ & 1 & 0 & 0 & 0 & 0 \\
\hline$S Q F$ & 12 & $\mathrm{~F}$ & $\mathrm{HL}$ & 0 & 2 & 0 & 1 & 0 \\
\hline WCV & 15 & M & $C D$ & 0 & 0 & 0 & 0 & 0 \\
\hline BFG & 12 & $\mathrm{~F}$ & HT & 2 & 0 & 0 & 0 & 0 \\
\hline MAG & 13 & $\mathrm{~F}$ & $\mathrm{HL}$ & 0 & 1 & 1 & 0 & 0 \\
\hline FSM & 17 & M & $\mathrm{HL}$ & 0 & 1 & 0 & 0 & 1 \\
\hline CBP & 18 & $M$ & $C D$ & 0 & 3 & 0 & 0 & 0 \\
\hline MKM & 13 & $\mathrm{~F}$ & $\mathrm{HT}$ & 1 & 0 & 0 & 0 & 0 \\
\hline CCP & 19 & $M$ & $\mathrm{HL}$ & 0 & 2 & 0 & 0 & 0 \\
\hline RTB & 15 & $\mathrm{~F}$ & $\mathrm{HL}$ & 1 & 0 & 0 & 0 & 1 \\
\hline YK & 13 & $\mathrm{~F}$ & $\mathrm{HL}$ & 1 & 1 & 0 & 1 & 0 \\
\hline DNAS & 15 & $\mathrm{~F}$ & HT & 0 & 1 & 0 & 1 & 0 \\
\hline MTM & 13 & $\mathrm{~F}$ & $C D$ & 0 & 0 & 0 & 1 & 0 \\
\hline CMR & 14 & $\mathrm{~F}$ & $C D$ & 3 & 0 & 0 & 0 & 0 \\
\hline CRB & 10 & $\mathrm{~F}$ & $\mathrm{HL}$ & 2 & 3 & 1 & 0 & 0 \\
\hline EAM & 19 & M & $\mathrm{HL}$ & 3 & 5 & 0 & 2 & 4 \\
\hline LVS & 17 & $\mathrm{~F}$ & $C D$ & 3 & 0 & 0 & 1 & 0 \\
\hline JPR & 13 & $\mathrm{~F}$ & HT & 0 & 1 & 1 & 1 & 0 \\
\hline LBR & 12 & $\mathrm{~F}$ & $\mathrm{HL}$ & 2 & 0 & 0 & 2 & 0 \\
\hline
\end{tabular}

M: masculino; F: feminino; CD: Cotrel-Dubousset; HL: Harrington-Luque; HT: Hartshill. 
intraoperatório, com média de 0,6 evento por cirurgia (Tabelas 3 e 4).

Comparando-se a resposta do PESS na pré-indução (valores basais) e no pós-operatório, em 1 (11,1\%) paciente do Grupo I houve uma diminuição maior que 50\% da amplitude de onda. No Grupo II, 6,2\% dos pacientes apresentaram uma alteração da amplitude maior que 50\% e $18,7 \%$ mantiveram uma latência maior de $3 \mathrm{~ms}$, (Tabelas 5 e 6$)$.

Em nenhum paciente deste estudo foi observado qualquer tipo de dano neurológico no pós-operatório.

\section{DISCUSSÃO}

A monitorização do potencial evocado somatosensorial (PESS) foi descrita pela primeira vez por Tamaki et al. ${ }^{9-7}$. Com esse método, a resposta somatosensorial é avaliada pelo seu valor basal (pré-indução) e periodicamente durante a cirurgia. Uma alteração intraoperatória, como a diminuição maior que $50 \%$ da amplitude e o aumento maior de $10 \%$ da latência de onda, é considerada sinal de disfunção da coluna posterior e um possível comprometimento medular ${ }^{8-11}$. A razão da realização da avaliação sensitiva decorre da proximidade dos tratos sensitivos e motores na medula, e uma lesão no trato motor indiretamente afetaria o trato sensitivo, alterando o PESS. Vale ressaltar que essa alteração é esperada quando a lesão é causada por um evento mecânico. Uma lesão vascular ou hipotensão intraoperatória (pressão arterial média menor de 60 $\mathrm{mmHg}$ ) pode causar alterações motoras sem alterações sensitivas concomitantes ${ }^{12,13}$.

Como dito, a anestesia representa um fator importante nessas cirurgias, podendo ser uma das causas de resultados falso-positivos, chegando, em alguns estudos, a uma taxa $4,5 \%{ }^{8}$. O procedimento anestésico foi realizado por via endovenosa com bombas de infusão

TABELA
intraoperatório
\begin{tabular}{lccc}
\multicolumn{4}{c}{ < Variação da latência da onda no } \\
\hline Grupo I & 4 & $>3 \mathrm{~ms}$ & Sem alterações \\
Grupo II & 6 & 5 & 5 \\
\hline
\end{tabular}

TABELA 5 - Relação entre a amplitude das ondas pós e pré-operatória (Amp pós $/ \mathrm{Amp}^{\text {pré}}$ )

\begin{tabular}{lccc}
\hline & $>\mathbf{5 0} \%$ & $<50 \%$ & Sem alterações \\
\hline Grupo I & 1 & 3 & 5 \\
Grupo II & 1 & 9 & 6 \\
\hline
\end{tabular}

TABELA 6 - Diferença entre o tempo de latência das ondas pós e pré-operatórias (Latpós - Latpré)

\begin{tabular}{lccc}
\hline & $>3 \mathrm{~ms}$ & $<3 \mathrm{~ms}$ & Sem alterações \\
\hline Grupo I & 0 & 3 & 6 \\
Grupo II & 3 & 5 & 8 \\
\hline
\end{tabular}

contínua, mantendo-se a condição mais estável possível do paciente. As medicações utilizadas foram preferencialmente o diprivan e o remi-fentanil, sem administração de medicações inalatórias. A manutenção da pressão arterial média de $65 \mathrm{mmHg}$ e a temperatura corporal de $36^{\circ}$ foi obtida durante a maior parte do procedimento cirúrgico ${ }^{14}$.

O objetivo deste estudo retrospectivo foi avaliar as alterações no PESS em diferentes técnicas de tratamento da escoliose idiopática. Assim como no estudo de Noonan et al. ${ }^{7}$, não houve resultados falso-negativos. Nenhum paciente apresentou déficits neurológicos no pós-operatório. No entanto, foi observada uma grande taxa de resultados falso-positivos (em torno de 14\%), diferentemente da literatura ${ }^{2,8,15,16}$. Uma possível explicação para o fato de esses pacientes não apresentarem déficits neurológicos, apesar da alteração presente no PESS, é a tendência a apresentarem uma depleção de volume intravascular ao final do procedimento cirúrgico, com a queda do hematócrito, decorrente da hemodiluição, pelo sangramento excessivo e o tempo cirúrgico prolongado, resultando em menor oxigenação da medula, podendo levar a alterações no PESS ${ }^{15}$.

As técnicas que utilizaram amarrilhas sublaminares (Grupo II) apresentaram com maior frequência alterações no PESS, podendo ser justificadas pela maior manipulação e exposição dos tecidos nervosos ${ }^{2,4,8}$. Uma maior exposição pode acarretar diminuição da temperatura local, e também a condição dos tecidos moles ao redor da coluna podem gerar uma alteração da condução do estímulo sensitivo $^{4,17}$. Um dos fatores limitadores deste trabalho foi a não-especificação, dentro dos dados colhidos, em relação ao tempo cirúrgico em que foram observados os abalos na monitorização do PESS, sendo os principais o momento da passagem do instrumental cirúrgico (Cotrel-Dubousset, sistema de Harrington-Luque e haste de Hartshill) a correção da deformidade.

Portanto, uma alteração no PESS pode representar uma lesão real, mas reversível da função medular. É recomendado que, ao se identificarem alterações na monitorização neurofisiológica da medula no período intraoperatório, alguma medida seja tomada, com uma interrupção temporária do ato cirúrgico, reavaliação dos parâmetros anestésicos e até mesmo retirada do instrumental ${ }^{15,18}$. Em caso de dúvida, deve ser realizado o teste do despertar (teste de Stagnara) para avaliação de déficit efetivo no paciente.

\section{CONCLUSÃO}

Há muitas questões acerca do método mais seguro para o tratamento cirúrgico da escoliose idiopática. Os procedimentos que utilizaram amarrilhas sublaminares apresentaram com maior frequência alterações no PESS, tanto no intraoperatório como ao final da cirurgia. No entanto, em nenhum paciente foi observado dano neurológico. A monitoração somatosensorial pode ser considerada um parâmetro precoce de lesão medular. 


\section{REFERÊNCIAS}

1. Cheng JC, Guo X, Sher AH, Chan YL, Metreweli C. Correlation between curve severity, somatosensory evoked potentials, and magnetic resonance imaging in adolescent idiopathic scoliosis. Spine. 1999;24(16):1679-84.

2. Roy EP 3rd, Gutmann L, Riggs JE, Jones ET, Byrd JA, Ringel RA. Intraoperative somatosensory evoked potential monitoring in scoliosis. Clin Orthop Relat Res. 1988;(229):94-8.

3. Nuwer MR, Dawson EG, Carlson LG, Kanim EA, Sherman JE. SEP spinal cord monitoring reduces neurologic deficits after scoliosis surgery: Results of a large multicenter survey. Electroencephalogr Clin Neurophysiol. 1995;96(1):6-11.

4. Luk KD, Hu Y, Wong YW, Leong JC. Variability of somatosensoryevoked potentials in different stages of scoliosis surgery. Spine. 1999;24(17):1799-804.

5. Hall JE, Levine CR, Sudhir $\mathrm{KG}$. Intraoperative awakening to monitor spinal cord function during Harrington instrumentation and spine fusion: description of procedure and report of three cases. J Bone Joint Surg Am. 1978;60(4):533-6.

6. More RC, Nuwer MR, Dawson EG. Cortical evoked potential monitoring during spinal surgery: Sesitivity specificity, reliability, and criteria for alarm. J Spinal Disord. 1988;1(1):75-80.

7. Tamaki T, Noguchi T, Takano H, Tsuji H, Nakagawa T, Imai K, Inoue S. Spinal cord monitoring as a clinical utilization of the spinal evoked potential. Clin Orthop Relat Res. 1984;(184):58-64.
8. Noonan KJ, Walker T, Feinberg JR, Nagel M, Didelot W, Lindseth R. Factors related to false- versus truepositive neuromonitoring changes in adolescent idiopathic scoliosis surgery. Spine. 2002;27(8):825-30.

9. Dawson EG, Sherman JE, Kanim LE, Nuwer MR. Spinal cord monitoring. Results of the Scoliosis Research Society and the European Spinal Deformity Society survey. Spine. 1991;16(8 Suppl):S361-4.

10. Wilson-Holden TJ, Padberg AM, Parkinson JD, Bridwell KH, Lenke LG, Bassett GS. A prospective comparison of neurogenic mixed evoked potential stimulation methods: utility of epidural elicitation during posterior spinal surgery. Spine. 2000;25(18): 2364-71.

11. York DH. A Critical Evaluation of the $50 \%$ Criterion in SEP Monitoring. Proceedings of the American Society of Neurophysiological Monitoring Annual Meeting, St. Louis, Missouri, 1995.

12. Kai Y, Owen JH, Lenke LG, Bridwell KH, Oakley DM, Sugioka Y. Use of sciatic neurogenic motor evoked potentials versus spinal potentials to predict earlyonset neurologic deficits when intervention is still possible during overdistraction. Spine. 1993;18(9):1134-9.

13. Owen JH. The application of intraoperative monitoring during surgery for spinal deformity. Spine. 1999;24(24):2649-62. Review.

14. Puertas EB, Wajchenberg M, Ferreira R, Scamardi FFR, Trandafilov Junior M. Comparison between the wake-up test and the intra-operative neurophysiologic monitoring with somato-sensitive evoked potentials in surgery for scoliosis. Coluna/ Columna. 2009;8(1):7-12.
15. Leung YL, Grevitt M, Henderson L, Smith J. Cord monitoring changes and segmental vessel ligation in the "at risk" cord during anterior spinal deformity surgery. Spine. 2005;30(16):1870-4.

16. Lesser RP, Raudzens P, Luders H, Nuwer MR, Goldie WD, Morris III $\mathrm{HH}$, et al. Postoperative neurological deficits may occur despite unchanged intraoperative SEP. Ann Neurol. 1986;19(1):22-5.

17. Loder RT, Thomson GJ, LaMont RL. Spinal cord monitoring in patients with nonidiopathic spinal deformities using somatosensory evoked potentials. Spine. 1991;16(12):1359-64.

18. Papastefanou SL, Henderson LM, Smith NJ, Webb JK. Surface electrode somatosensory evoked potentials in spinal surgery implications for indications and practice. Spine. 2000;25(19): 2467-72.

\section{Correspondência:}

Renato Hiroshi Salvioni Ueta

Rua Borges Lagoa, 783, $5^{\circ}$ andar - Vila Clementino

CEP: 04038-003 - São Paulo (SP), Brasil

Tel.: (1 1) 5571-6621

E-mail:salvioniveta@uol.com.br 